\title{
Microplastic Fibers Released by Textile Laundry: A New Analytical Approach for the Determination of Fibers in Effluents
}

\author{
Jasmin Haap ${ }^{1, *}$, Edith Classen ${ }^{1}$, Jan Beringer ${ }^{1}$, Stefan Mecheels ${ }^{1}$ and Jochen S. Gutmann ${ }^{2,3}$ (D) \\ 1 Hohenstein Institut für Textilinnovation gGmbH, Schlosssteige 1, 74357 Bönnigheim, Germany; \\ e.classen@hohenstein.de (E.C.); j.beringer@hohenstein.de (J.B.); s.mecheels@hohenstein.de (S.M.) \\ 2 University Duisburg-Essen, Institute of Physical Chemistry and Center for Nanointegration, Duisburg-Essen, \\ Universitätsstraße 5, 45117 Essen, Germany; jochen.gutmann@uni-due.de \\ 3 Deutsches Textilforschungszentrum Nord-West gGmbH, Adlerstr. 1, 47798 Krefeld, Germany \\ * Correspondence: j.haap@hohenstein.de
}

Received: 19 August 2019; Accepted: 25 September 2019; Published: 7 October 2019

\begin{abstract}
The detection of shedded fibers in effluents from textile washing has attracted much attention due to its reported contribution to microplastic pollution. Commonly used analytical methods for fiber detection in liquids are based on filtration with subsequent microscopic analysis and/or gravimetric weighing. These approaches are time-consuming and prone to errors. In this study, an approach based on dynamic image analysis was applied in order to set up an efficient method to analyze fibers in effluents from washing processes. In an initial validation step, reliable information on the counts of fibers and the morphological characteristics were confirmed. For wastewaters from polyester-cotton blends, the chemical nature of the fiber debris (natural vs. synthetic origin) was determined by combining the dynamic image analysis with a chemical pre-treatment. In this study, dynamic image analysis was revealed as a rapid, non-destructive, precise, and reliable technology for the characterization and quantification of the fiber debris, offering a promising approach for fiber analysis in liquids.
\end{abstract}

Keywords: microplastic; textile fibers; laundry; analytical method; fiber shedding; dynamic image analysis

\section{Introduction}

The accumulation of anthropogenic debris in the environment has raised growing concern. The presence of microplastic (synthetic particles and fibers $<5 \mathrm{~mm}$ ) was confirmed in marine habitats, freshwater ecosystems, air as well as in soil [1-4]. Microplastics found in these compartments were heterogeneous in size, shape and chemical composition [5]. Fibers are one of the most frequent particle shapes of microplastics detected in environmental samples [6,7]. Research groups worldwide reported on the uptake of synthetic fibers by various aquatic species and animals in terrestrial ecosystems [8,9].

Textiles have been identified as a major source of microplastic as they have the potential to release fibers during production, their use phase (wearing, washing and drying) and disposal [10-13]. Shedded fibers can enter nature via direct pathways, however, the presence of synthetic fibers in the environment is mostly attributed to the laundry process [13,14]. During washing, the textiles are exposed to different strains which may cause fibers to be released into the washing effluent [15]. Particularly, the four Sinner's parameters: washing chemistry, temperature, washing duration and mechanical stress may contribute to fiber emissions [15,16]. Further, the type of washing machine, including aspects of axis direction (front/top load), machine capacity, rotation and spin dry rate and amount of water used can affect the mass loss of textiles [16-18]. Additionally, the apparel design 
including the textile characteristics, e.g., fiber type, yarn construction, and surface treatment have considerable potential to impact fiber release [15,18].

To date, there is no effective way to remove fibers from the wastewater of washing processes as the lint filters in the washing machines are not designed to totally hold back the entire particle/fiber discharge [19]. Furthermore, studies have shown that wastewater treatment plants have a limited retention capacity for microplastic particles and fibers [20,21].

To tackle the issue of fiber release, different analytical methodologies have been developed. Filtration of the wastewater after laundering with subsequent manual counting of the fibers recovered on a filter material by microscopy was the method of choice of different research groups $[10,15,17,22]$. Quantitative determinations were either performed by scanning electron microscopy (SEM-) imaging or light microscopy $[10,17,22]$. Fiber counting was mostly applied manually by the naked eye, which implies that these procedures are highly time-consuming. Improvements have been developed by De Falco et al. counting fibers on SEM-images acquired in orthogonal direction of the filter and extrapolating to the complete surface [22]. Jönsson et al. applied an automated software-based fiber counting of the filter surface using microscopy [23]. However, visual counting methods entail the risk of uncertainty. An overload of fibers on the filter may lead to an overlapping of individual fibers $[15,23]$. Consequently, the number of fibers is potentially underestimated. For this reason, a homogeneous distribution of the fibers on the filter surface has to be ensured [15].

Gravimetric analysis of the filtered material is a further approach reported in literature [16-18,24]. The fibers are separated from the liquid mechanically through a filter, usually added by a vacuum pump, and the mass of the recovered weight is determined. For both the counting and the weight analysis, the selection of the filter pore size impacts the results significantly. Small pore sizes increase the retention capacity of fibers, however, clogging effects are more likely to occur [24]. In two studies, cascade filtering was applied to prevent filter blockage $[18,23]$. Further, the selection of an appropriate filter material, the sample handling in terms of drying, conditioning, and weighting factors potentially influence the quantitative results. Furthermore, material loss as well as side contaminations are critical points and imply a strong need for repeated negative control tests [25].

Gravimetric evaluations are comparatively straightforward-however they do not provide information about morphological properties and dimensions of the fibers. These characteristics are important pointers for causal researches in terms of fiber release and risk assessments [26]. The lack of visualization leads to the assumption that the entire material recovered on the filter is attributed to fibers only. However, studies confirmed the presence of fibers and particles from non-textile sources in wastewaters from laundry $[23,27]$. In this sense textiles are A) an emitting source of fibers as fiber shedding occurs, and B) serve as a carrier for adhered foreign fibers and particles of various materials, shapes and sources. In the latter case dust, production residues or contaminants from the surrounding are examples of fibers and particles adhering to the textile surface and may be released in the washing process $[23,27]$. Consequently, there is a significant risk of overestimating the results obtained by weight analysis.

Most of the studies available reported a fiber release during domestic laundry, only a minor number of publications investigated industrial washing processes in their research [15,22].

At present, a comparability of different studies is hindered by the diversity of analytical technologies and pretreatment procedures as well as the diversity of selected textiles and applied washing processes [19]. Establishing a harmonized protocol for the analysis of fibers in wastewaters from textile laundry is a growing need to generate a comprehensive understanding of fiber abrasion during washing and to develop feasible solutions.

Textiles from the clothing sector are the most commonly washed items. Typically, these textiles are made out of five main polymer types: polyester, cotton, polyamide, wool and silk [28]. In the majority of studies, synthetic textiles made out of polyester were selected for the investigations $[10,15,22,23]$. Although polyester-cotton blends reflect the most commonly manufactured fiber composition in the clothing industry, there is a lack of information regarding the shedding behaviour of fiber mixtures 
during laundry [29]. Napper et al. analyzed the wastewaster effluent of polyester-cotton garments, however, they determined the total amount of fibers, not distinguishing between synthetic and natural source [16].

In this study, we evaluated the applicability of dynamic image analysis for the analysis of wastewater samples from textile laundry. Dynamic image analysis is an optical detection system for particle characterization commonly applied in pharmacy, the food industry, and geology [30-32].

The measuring system allows an online detection of particles in solid state or particles suspended in liquids without specific sample pretreatment. In the case of liquid analysis, the well-dispersed effluent is transported through a cuvette and a high-resolution camera captures images of the particles in the image plane. Motion blur during image acquisition is minimized by using a pulsed laser as a light source with an exposure time of approximately $1 \mathrm{~ns}$. Sophisticated image analysis algorithms enable statistical analysis regarding the fiber number and morphological characteristics, e.g., length, diameter, straightness, and elongation. The repeatability and accuracy of the analytical method was investigated using single component fiber systems. The number of fibers per measurement was compared to the results of commonly applied microscopic methods. In addition, the composition of the fiber containing effluent of a polyester-cotton intimate blend was examined regarding the content of cotton and polyester. For this purpose, the dynamic image analysis was combined with a sulphuric acid pretreatment, which enables a full hydrolysis of the cotton component prior to the measurement.

\section{Materials and Methods}

\subsection{Fibers}

Polyamide 6.6 fibers with a length of $0.3 \mathrm{~mm}$ and a diameter of $15 \mu \mathrm{m}$ (1.7 dtex fiber fines), referred as to reference fibers, obtained from Borchert + Moller GmbH were used for the validation. The scanning electron microscopy image in Figure 1 shows the morphology of the fibers in detail.

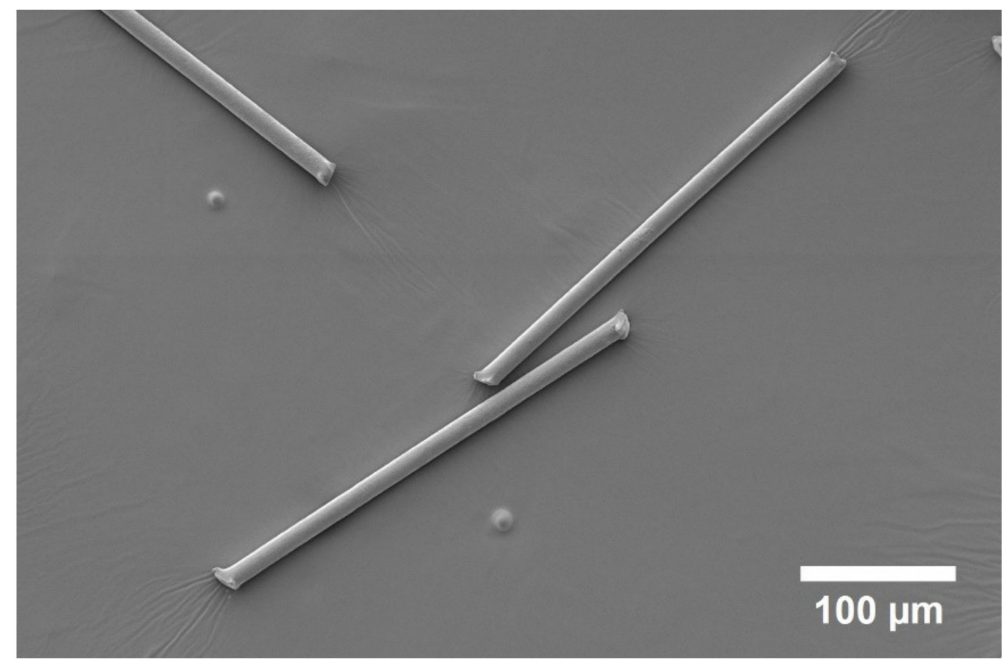

Figure 1. Scanning electron microscopy image of the polyamide 6.6 fibers $(0.3 \mathrm{~mm}, 1.7 \mathrm{dtex})$ showing highly size-uniform and straight fibers.

For quantitative fiber analysis aqueous suspensions of the uniform reference material were prepared. $3.15 \mathrm{mg}$ and $6.17 \mathrm{mg}$ fibers were suspended in $500 \mathrm{~mL}$ detergent/water solution respectively.

\subsection{Fabric}

For the washing experiments a workwear fabric made out of 50/50 polyester-cotton (PES/CO) was selected. The woven fabric (S-twill 2/1) comprised of an intimate mixture (warp: 65/35\% PES/CO, weft: $35 / 65 \% \mathrm{PES} / \mathrm{CO}$ ) of staple yarn with a surface weight of $215 \mathrm{~g} / \mathrm{m}^{2}$ and yarn count warp: $40 \mathrm{Nm}$, weft: $24 \mathrm{Nm}$. 


\subsection{Contamination Prevention}

To minimize the risk of sample contamination the working space was cleaned thoroughly using lint-free wipes. All laboratory glassware was cleaned prior to use, oven dried and stored in a dust-free environment. Glassware was kept covered to prevent airborne contaminations. In contrast to reported studies, no cotton lab coat was used as personal laboratory equipment. Instead, a labcoat made of a polyamide monofilament commonly used for surgical gowns was worn as it is designed to have a low-lint surface. Additionally, arm sleeves made of polyethylene foil and gloves were worn as preventative measure. Ultrapure water $(0.055 \mu \mathrm{S} / \mathrm{cm})$ from a TKA-GenPure system (Thermo Scientific) with an additional $0.2 \mu \mathrm{m}$ particle filter was used to ensure particle and fiber free water quality. Negative controls were applied regularly.

\subsection{Pretreatment of Fabrics}

The fabrics were pre-washed according to EN ISO 15797:2004 to remove non-permanent textile auxiliaries and residues from production. Deviating from the ISO standard no additional textile ballast was added to avoid cross contamination of the samples. The fabric was cut to $170 \mathrm{~mm} \times 165 \mathrm{~mm}$ pieces, cleaned with a lint roller on the inner side and folded once in the half. The final sample size was $85 \mathrm{~mm} \times 165 \mathrm{~mm}$. Using a textile glue (Skinotex, Weniger) the corners and edges were sealed to prevent fiber loss. For this purpose, the textile was immersed into the adhesive by $0.5 \mathrm{~mm}$ on each side. The prepared specimens were air dried overnight in a dust free environment prior to washing.

\subsection{Washing Procedure}

The washing experiments were carried out using a laboratory washing test for industrial laundry processes based on the ISO 105-C12:2004 for colorfastness analysis in industrial washing processes with some modifications. This test is designed to mimic the mechanical stress of 5-10 washing cycles (in relation to color fastness) in an industrial washing machine.

A lab-scale washing machine "Labomat" (BFA, Werner Mathis AG) equipped with $8 \times 500 \mathrm{~mL}$ steel canisters was used to launder the fabric samples. For these experiments two fabric samples per canister were used. The laboratory washing machine was operated under controlled conditions. The turning velocity was set to $40 \pm 2 \mathrm{rpm}$ with a reversing time of 30 seconds (left and right turn) and the mechanical stress was simulated by 50 stainless steel balls with a diameter of $6 \mathrm{~mm}$. The experiments were performed at $40 \pm 2{ }^{\circ} \mathrm{C}$ with a heating rate of $1.5^{\circ} \mathrm{C} / \mathrm{min}$ and a washing duration of $30 \mathrm{~min}$ in total, including the heating period. Then, $160 \mathrm{~mL}$ washing liquor containing $2 \mathrm{~g} / \mathrm{l}$ liquid color detergent (Derval Rent, Kreussler, pH = 11) without optical brightener applied for industrial purposes was used. The washing experiment was repeated three times.

After washing, the samples were removed and rinsed with ultrapure water from both sides using a wash bottle. The steel balls were separated from the washing liquid by a strainer and rinsed with ultrapure water. All washing liquids were collected, and the solution was diluted to a total volume of $300 \mathrm{~mL}$ prior dynamic image analysis measurements.

\subsection{Chemical Separation of Polyester and Cotton Fibers}

The samples were treated with sulphuric acid based on DIN EN ISO 1833-11 to achieve a chemical separation of polyester and cotton. Using the sulphuric acid treatment the cotton component is hydrolyzed, whilst polyester is left over.

The fabric was cut to $3 \mathrm{~cm} \times 3 \mathrm{~cm}$ pieces and conditioned at $20 \pm 2{ }^{\circ} \mathrm{C}$ and $65 \pm 4 \%$ humidity prior to weight analysis. The specimens were incubated in $50 \mathrm{~mL}$ concentrated sulphuric acid (75\% p.a., $\mathrm{KB}$ Bernd Kraft) for $1 \mathrm{~h}$ at $50^{\circ} \mathrm{C}$. Both fabric sides were thoroughly rinsed with water and neutralized with

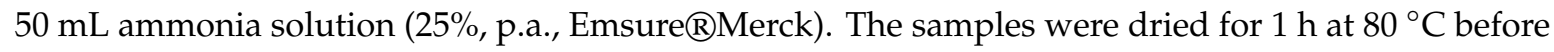
conditioning in the climate chamber. The mass loss was determined. 
In the case of the wastewaters, the suspensions were filtered through a cellulosic filter (Whatman $囚$ grade 40 ) with a pore size of $8 \mu \mathrm{m}$ and a diameter of $90 \mathrm{~mm}$ under vacuum conditions. The filter was carefully rinsed by $100 \mathrm{~mL}$ ultrapure water to remove excess detergent and left to dried for $15 \mathrm{~min}$ at $50^{\circ} \mathrm{C}$ in a glass Petri dish. Then, $20 \mathrm{~mL}$ sulphuric acid was added and the suspension was incubated for $1 \mathrm{~h}$ at $50^{\circ} \mathrm{C}$. The samples were carefully shaken in regular time intervals of approximately $20 \mathrm{~min}$. After the treatment, the acid solution was neutralized. The entire liquid was transferred to $150 \mathrm{~mL}$ water while stirring and the ammonia solution was added until a $\mathrm{pH}$-value of 7 was reached. Each sample was diluted to a total volume of $300 \mathrm{~mL}$ and cooled down to room temperature prior to analysis.

\subsection{Dynamic Image Analysis (DIA)}

A dynamic image analysis device (Qicpic, Sympatec $\mathrm{GmbH}$ ) was applied for the fiber detection. Windox 5.10.0.3 software was used to acquire and analyze the data. The measuring system was equipped with a beaker containing an outlet tap on the bottom of the flask. This setup was used as a reservoir for the wastewater samples and a magnetic stirrer was installed to prevent sedimentation. A peristaltic pump (MCP Process with pump head SB 3V, Ismatec) connected the reservoir and the flow-through cuvette of the detection system $(0.2 \mathrm{~mm}$ thickness). Pulsation during liquid transportation was reduced by a self-made pulsation dampener consisting of a T-piece adapter and a tube of $30 \mathrm{~cm}$ length. In order to obtain an air pocket for appropriate dampening function the end of the tube was sealed. The volume flow of the pump was set to $172 \mathrm{~mL} / \mathrm{min}$ and image acquisition was performed at a frame rate of $85 \mathrm{fps}$. Before starting the measurement, the liquid was circulated (drainage beaker $\rightleftharpoons$ measuring system) to flush the system. The liquid was magnetically stirred repeatedly for $5 \mathrm{~s}$ within intervals of $30 \mathrm{~s}$. The measurement was started and after a lead time of $3 \mathrm{~s}$ the tube was laid from the drainage beaker into a collecting vessel. After the cuvette was fully drained with liquid, the measurement was stopped. Each sample was analyzed two times. The images were converted into binary images by the integrated software. Eventually, fiber doublings which occurred due to inhomogeneous volume flow events were subtracted manually from the counting results. For the evaluation process a minimum fiber length of $50 \mu \mathrm{m}$ and minimum diameter of $7 \mu \mathrm{m}$ were defined. Additionally, the shape parameters straightness, convexity and aspect ratio were included in the evaluation process.

The fiber quantification by dynamic image analysis (DIA) is limited to an indirect measurement of the total fiber count according to the measuring set up. Figure 2 shows a schematically cross-section of the flow-through cuvette. The cuvette is comprised of a circular glass window ( $3.4 \mathrm{~cm}$ in diameter) and the total horizontal axis is $4.1 \mathrm{~cm}$ in length. The cuvette width can be divided in eleven equally sized detection frames. Each square is $3.75 \mathrm{~mm} \times 3.75 \mathrm{~mm}$ and corresponds to the dimensions of the detector size. The detection volume is $2.9 \mu \mathrm{L}$ respectively. The measurement is usually performed at the cuvette center (position 0). However, the setup allows the cuvette position to be changed in one direction. The colored areas are the possible measuring positions and the negative position numbers indicate the detection focus moving direction starting from the central point position 0 . Gauge blocks were used to ensure a defined cuvette movement in $3.75 \mathrm{~mm}$ steps. Fiber analysis was performed for cuvette position $0-3$. Position 4 is outside the detection area and, likewise, position 5 is not detectable likewise as this frame is located out of the cuvette window.

\subsection{Microscopy}

The reference fibers were analyzed using a scanning electron microscope (JSM-5610 LV, Jeol). The dry fibers were mounted on an adhesive tape and coated with gold under vacuum conditions before imaging to prevent electrostatic charging of the surface (Cressington Sputtercoater 108 auto, $40 \mathrm{~mA},<0.1 \mathrm{mbar}, 90 \mathrm{~s}$ ). SEM observations and image acquisition were performed using an accelerating voltage of $1-2.5 \mathrm{kV}$. Fiber length and diameter was determined by the freeware image processing software ImageJ (release $1.49 \mathrm{v}$ ). 
Fiber counting in suspensions were additionally performed by visual microscopy using a digital microscope (VHX-S15, Keyence). For this, $50 \mu \mathrm{L}$ aliquots were withdrawn from the homogenized model wastewater sample and the number of fibers within this volume was counted.

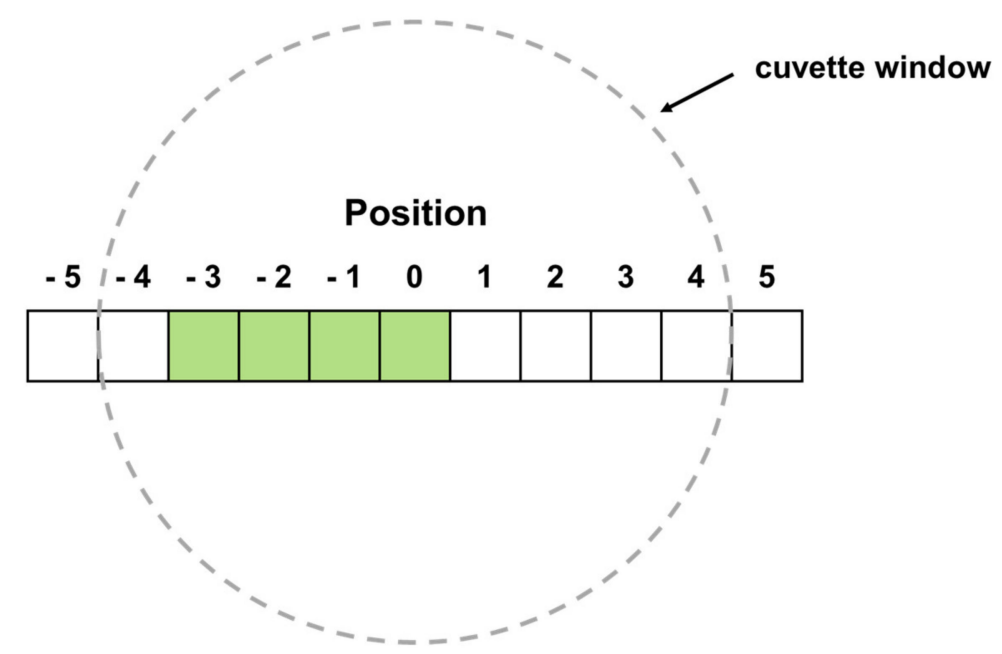

Figure 2. Cross-section of the dynamic image analysis flow-through cuvette. The horizontal axis is divided into $3.75 \mathrm{~mm} \times 3.75 \mathrm{~mm}$ frames corresponding to the detection window. Positions 0 to -3 (green boxes) represent the experimentally accessible areas, whereas the remaining frames are not usable due to setup limitations.

\section{Results and Discussion}

\subsection{Validation}

\subsubsection{Morphological Properties}

Fiber length and diameter results of the $0.3 \mathrm{~mm}$ polyamide 6.6 reference fibers obtained by dynamic image analysis (DIA) were compared with scanning electron microscopy (SEM-) images. The latter technique is widely accepted for particle characterization and has been validated by standards for size determinations. If the optical setup and data processing algorithm used by the dynamic image analysis was appropriate, then the particle size distribution should be in good alignment with the ones obtained from SEM-imaging. Figure 3 shows the comparison of the fiber length distribution. The average fiber length analyzed by SEM was $297 \pm 41 \mu \mathrm{m}$, the length of fiber obtained by DIA $325 \pm 45 \mu \mathrm{m}$. The diameter was found to be $14 \pm 1 \mu \mathrm{m}$ for SEM and $16 \pm 2 \mu \mathrm{m}$ for DIA. For both morphological parameters, the results are in accordance within the standard deviation. The size distribution referring to the fiber length (Figure 3) appears to be slightly broader when analyzed by SEM. However, this variation might be attributed to the analytical data processing. Whilst a total number of $>5000$ fibers was detected by DIA measurements within a single measurement and a time frame of a few minutes, a much smaller amount of approximately 70 fibers were analyzed by SEM-imaging due to the manual measuring effort of hours. Overall, the data obtained from both measurement techniques align well, indicating that dynamic image analysis provides accurate data in terms of length and diameter fiber dimensions.

\subsubsection{Quantitative Validation}

The data presented so far have demonstrated that the dynamic image analysis is a suitable tool for characterizing the morphological properties of length and diameter of polyamide 6.6 reference fibers in liquids. Since there is no data about the application of dynamic image analysis for fiber quantification reported to our knowledge this was challenged in this section. 


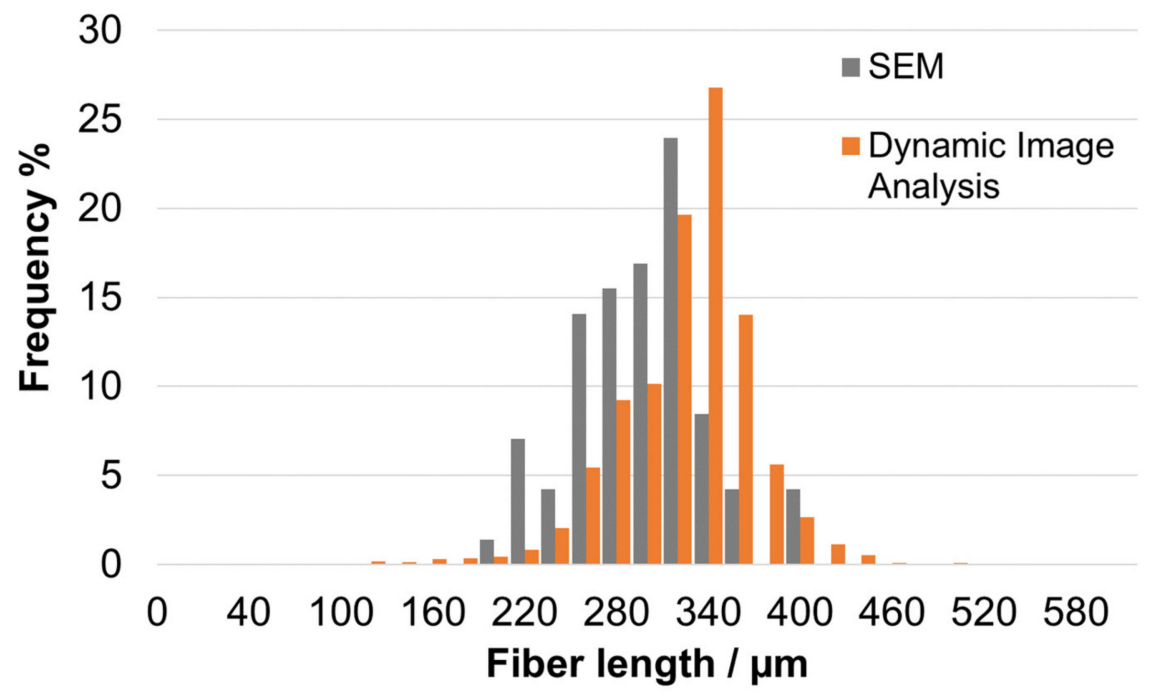

Figure 3. Distribution of the fiber length $(0.3 \mathrm{~mm}$ polyamide 6.6 reference fibers) determined by scanning electron microscopy (SEM, grey columns) and dynamic image analysis (orange columns). A total number of 70 fibers was analyzed by SEM-imaging whilst $>5000$ fibers were detected by a single dynamic image analysis measurement.

The dynamic image analysis only allows the analysis of a limited detection volume during a single measurement. Consequently, the data needs to be extrapolated to the entire sample volume. To study the effects occurring when micro-sized fibers are probed at different detection positions of the dynamic image analysis cuvette a suspension of reference fibers was used.

Figure 4 presents the amount of fibers obtained from dynamic image analysis in correlation to the cuvette position. The highest amounts of fibers were measured in the central positions 0 and 1 of the cuvette. The farther the detection focus is shifted away from the center, the lower the number of fibers measured. For the positions 0 to -3 three replicates were analyzed. Data points of positions -4 and -5 were calculated. Due to the axial symmetry of the cuvette, a symmetric liquid flow was assumed. The distribution of the fiber amounts correlating to the measuring position indicates a flow rate distribution within the cuvette matching a parabolic flow profile. Shear stress influences the fluid dynamics causing a lower fluid velocity at the cuvette wall regions and leading to a decrease in fiber amount counted on the outer sides (laminar flow). The available data allow to establish a correlation between the measuring position and fiber counts. Based on these experimental results a calculation formula was derived that permits to quantify the total fiber amount in a suspension by one single dynamic image analysis measurement at the cuvette center.

The validity of the calculation concept was verified by a method comparison performed with two fiber suspensions, $3.15 \mathrm{mg}$ (S1) and $6.17 \mathrm{mg}$ (S2) reference fibers respectively. Figure 5 shows the fiber values obtained by three different measurement methods; calculation of theoretical fiber amount, dynamic image analysis, microscopy. The calculation of the expected fiber number is based on the average fiber characteristics length and diameter (S1: length $328 \pm 46 \mu \mathrm{m}$, diameter $17 \pm 2 \mu \mathrm{m}$, S2: length $327 \pm 46 \mu \mathrm{m}$, diameter $16 \pm 2 \mu \mathrm{m}$ ) determined by dynamic image analysis from four replicate DIA measurements and the density of polyamide $6.6\left(1.14 \mathrm{~g} / \mathrm{cm}^{3}\right)$ [33]. Dynamic image analysis measurements were applied following the measuring and calculation procedure described previously. As a third comparative method, the number of fibers was determined visually using optical microscopy. Overall, the data showed that the results obtained by all three methods aligned quantitatively. This pattern was robust when doubling the fiber amount. 


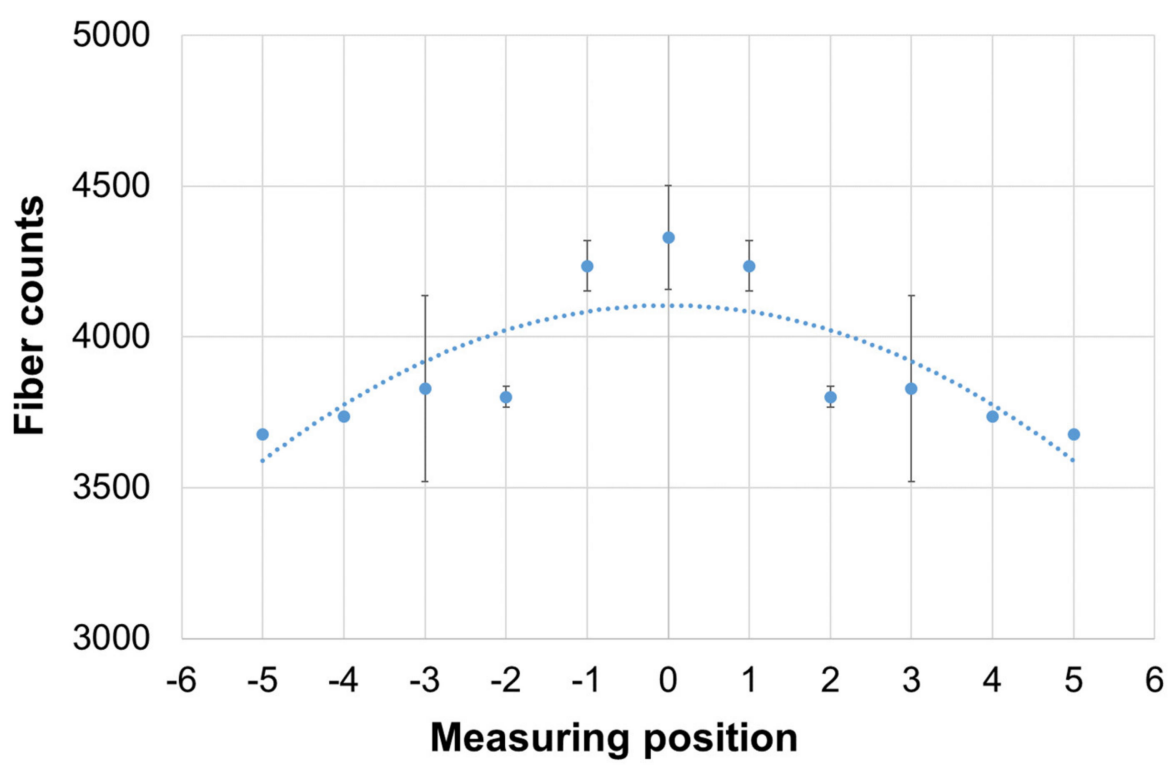

Figure 4. Fiber numbers determined by dynamic image analysis in correlation to the measuring position of the cuvette on horizontal axis. Fiber counts of position -4 and -5 are calculated values. A symmetric fiber distribution was assumed, and a quadratic curve fitting was applied.

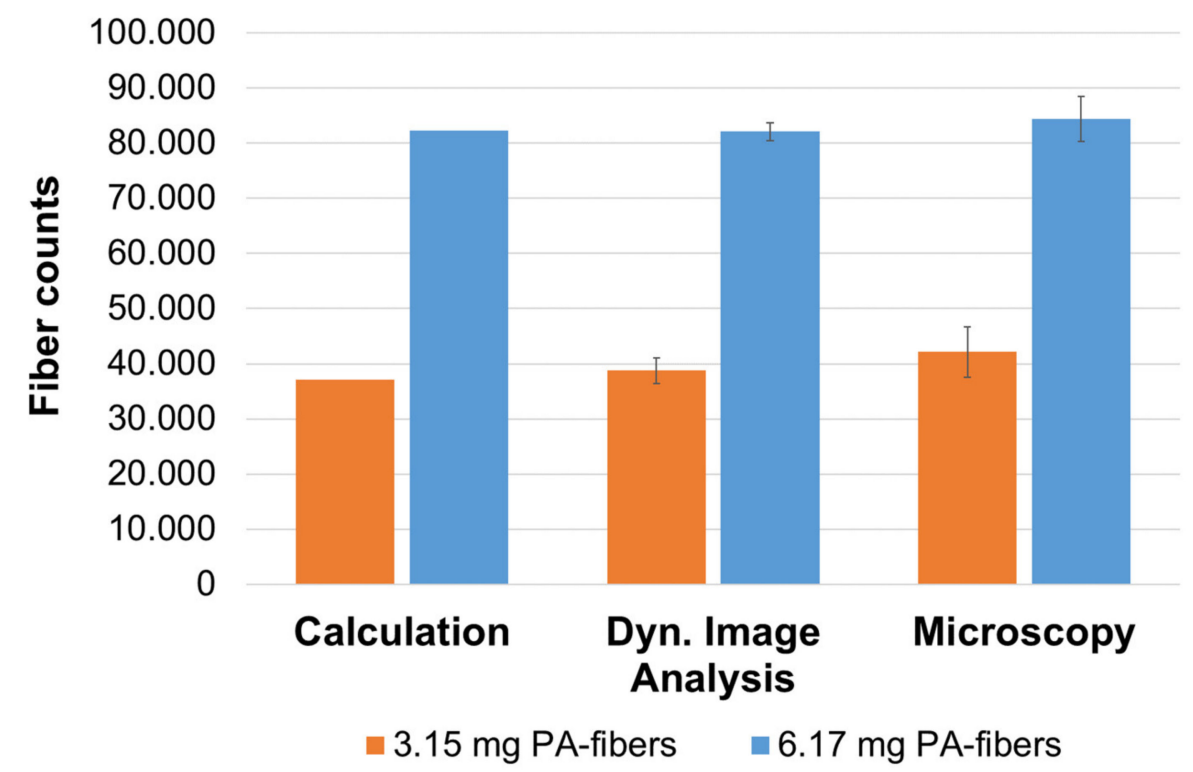

Figure 5. Results of the quantitative fiber determination of suspended polyamide 6.6 (PA-) fibers (0.3 mm length, $1.7 \mathrm{dtex}$ ). The fiber counts were determined for 3.15 and $6.17 \mathrm{mg}$ fiber suspensions respectively by three methods: calculation of the theoretical number of fibers, dynamic image analysis and optical microscopy counting.

The main differences between the dynamic image analysis and static microscopy were observed during the data acquisition process. While the microscopic determination requires a measuring effort of hours, the exact same results of the dynamic image analysis measurements were obtained within a few minutes. The reason for the time-consuming microscopy analysis is the manual counting procedure and the small area that is visually accessible each round [23]. Besides the time factor, microscopic analysis is also prone to errors and subjective assessment of the experimenter. Dekiff et al. observed in their study that three persons analyzing the same sample by using the same optical method received three different results [34]. Additionally, microscopic measurements require a homogeneous and a representative distribution of the fibers on the substrate $[15,23]$. A high fiber content entails the risk 
of overlapping and crossing fibers. This increases the chance of underestimating the total number of fibers in the samples [15]. To overcome this issue adapted sample preparation procedures are required. Jönsson et al., for example, applied a cascade filtering procedure prior to microscopy [23]. However, it should be kept in mind that any additional step increases the complexity and may influence the uncertainty and the error of the analytical method. In contrast, no specific sample pretreatment is needed for the dynamic image analysis. In case of high fiber loads a homogeneous distribution during measurement can be achieved by a simple dilution combined with sufficient stirring of the analyzed wastewater sample. Furthermore, dynamic image analysis measurement is non-destructive and the results are not influenced by subjective evaluations of the experimenter.

\subsection{Chemical Fiber Separation and Identification}

A 50/50 polyester-cotton fabric was chosen for evaluating the shedding behavior of both fiber types during laundering. Separating polyester and cotton fibers from a mixture by concentrated sulphuric acid was investigated first conducting the experiments on the textile surface. If the chemical treatment conditions were appropriate a degradation of the cotton component was expected. Figure 6 shows the textile surface characterization before and after sulphuric acid treatment. Indeed, surface changes were observed after chemical incubation, indicating a more aligned structure in the SEM-image. Besides optical changes, the haptic properties turned to a smoother surface. Both observations point out characteristic properties of synthetic fibers. This result was consistent with the weight loss of $47 \pm 1 \%$ concluding that the cotton component was fully removed. As a control experiment a 100\% polyester fabric was exposed to sulphuric acid. In fact, no mass loss was measurable and no changes in surface structure were observed. These experiments confirmed the hydrolysis of cotton under sulphuric acid incubation whilst polyester remains unaffected.
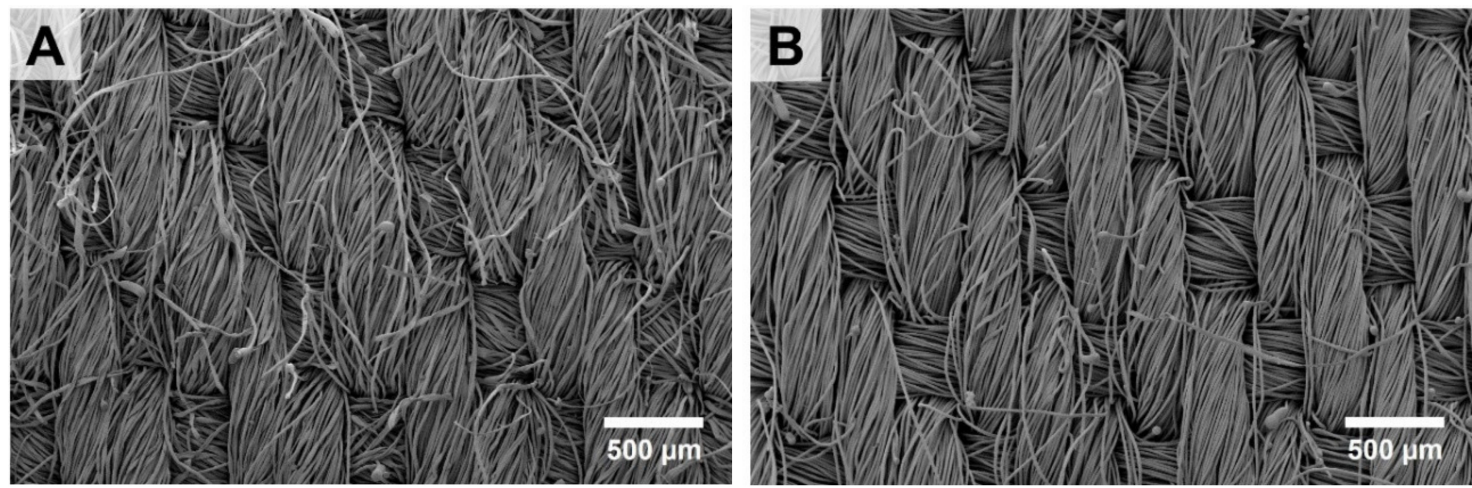

Figure 6. SEM-images of a 50/50 polyester-cotton intimate blended fabric. (A) Surface of the untreated fabric (B) Fabric surface after treatment with concentrated sulphuric acid. It shows a more aligned structure and a smoother surface after sulphuric acid incubation, confirming the removal of cotton.

In a further step, the wastewater from laundering the fabric was investigated regarding the composition of the fiber debris. For this purpose, the optical detection method (DIA) was combined with the sulphuric acid treatment. Figure 7 shows the fiber counts for cotton and polyester fibers in wastewater. Expressing the results in percentage, the proportion of released fibers is $14 \pm 3 \%$ polyester and $86 \pm 3 \%$ cotton. In this case, the amount of cotton fibers is six times higher compared to the amount of polyester fibers in the mixture.

To date, there is no data available that quantified the individual proportion of polyester and cotton fibers in the wastewater of polyester/cotton blends. Existing studies focusing on the shedding behavior of polyester/cotton blends did not distinguish between the fiber types [16,35]. Instead, the total number of fibers were evaluated. However, the observations in this study can be attributed to differences in the shedding behavior. In fact, synthetic fibers are generally considered to have a higher abrasion resistance compared to natural fibers [36]. 


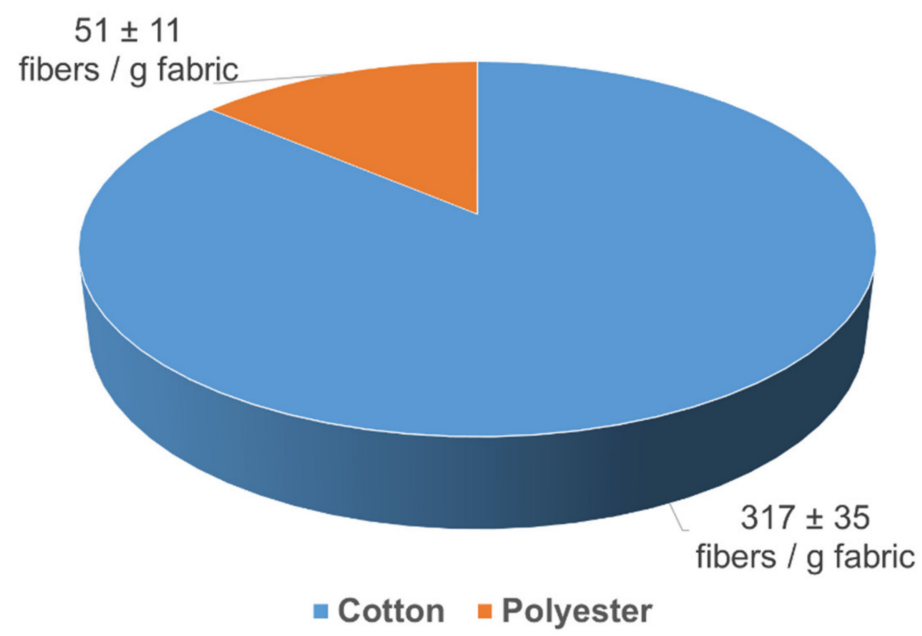

Figure 7. Fiber counts per $\mathrm{g}$ fabric of polyester and cotton fibers regarding the total amount of shedded fibers. These results refer to a wastewater sample from a washing process of a 50/50 polyester-cotton intimate blended fabric analyzed by dynamic image analysis.

\section{Conclusions}

In this study, dynamic image analysis has been introduced as an alternative instrumental method for fiber characterization and quantification in wastewater from laundry processes. Analytical method validation was conducted by comparing fiber morphology and quantitative analysis of dynamic image analysis with conventional optical microscopy methods. Using a single-component fiber system, good data agreement was observed in all experiments.

The main advantages of the dynamic image analysis presented in this study compared to commonly applied analytics could be confirmed as:

- measurement of a larger data set for high numbers of fibers within a measuring time of few minutes

- software automated evaluation of quantitative results and morphological properties

- $\quad$ non-destructive analysis

- lack of elaborated sample-pretreatment.

Finally, combining the dynamic image analysis with the sulphuric acid treatment provided quantitative information on the chemical composition of polyester-cotton fiber debris.

The capability of dynamic image analysis to simultaneously measure fiber morphology characteristics and fiber counts within short measuring times could help to provide valuable insights into a variety of textile applications with regard to fiber release and support new product and process developments.

Author Contributions: Conceptualization, J.H.; Investigation, J.H; Methodology, J.H.; Project administration, J.H.; Validation, J.H.; Writing—original draft, J.H.; Writing—review \& editing, E.C., J.B., S.M., J.S.G.

Funding: The IGF research project no $19219 \mathrm{~N}$ of the research association Forschungskuratorium Textil e.V., Berlin/Germany was funded via the AiF within the framework of the program for funding the industrial cooperative research and development (IGF) by the German Federal Ministry of Economic Affairs and Energy (BMWi) based on a decision of the German Federal Parliament.

Acknowledgments: We acknowledge support by the Open Access Publication Fund of the University of Duisburg-Essen.

Conflicts of Interest: The authors declare no conflict of interest.

\section{References}

1. Gasperi, J.; Wright, S.L.; Dris, R.; Collard, F.; Mandin, C.; Guerrouache, M.; Langlois, V.; Kelly, F.J; Tassin, B. Microplastics in air: Are we breathing it in? Curr. Opin. Environ. Sci. Health 2018, 1, 1-5. [CrossRef] 
2. De Souza Machado, A.A.; Kloas, W.; Zarfl, C.; Hempel, S.; Rillig, M.C. Microplastics as an emerging threat to terrestrial ecosystems. Glob. Change Biol. 2018, 24, 1405-1416. [CrossRef] [PubMed]

3. Eerkes-Medrano, D.; Thompson, R.C.; Aldridge, D.C. Microplastics in freshwater systems: A review of the emerging threats, identification of knowledge gaps and prioritisation of research needs. Water Res. 2015, 75, 63-82. [CrossRef] [PubMed]

4. Gall, S.C.; Thompson, R.C. The impact of debris on marine life. Mar. Pollut. Bull. 2015, 92, 170-179. [CrossRef] [PubMed]

5. Lambert, S.; Scherer, C.; Wagner, M. Ecotoxicity testing of microplastics: Considering the heterogeneity of physicochemical properties. Integr. Environ. Assess. Manag. 2017, 13, 470-475. [CrossRef]

6. Zhang, G.S.; Liu, Y.F. The distribution of microplastics in soil aggregate fractions in southwestern China. Sci. Total Environ. 2018, 642, 12-20. [CrossRef]

7. Naji, A.; Esmaili, Z.; Mason, S.A.; Vethaak, A.D. The occurrence of microplastic contamination in littoral sediments of the Persian Gulf, Iran. Environ. Sci. Pollut. Res. 2017, 24, 20459-20468. [CrossRef]

8. Zhao, S.; Zhu, L.; Li, D. Microscopic anthropogenic litter in terrestrial birds from Shanghai, China: Not only plastics but also natural fibers. Sci. Total Environ. 2016, 550, 1110-1115. [CrossRef]

9. Rochman, C.M.; Tahir, A.; Williams, S.L.; Baxa, D.V.; Lam, R.; Miller, J.T.; Teh, F.-C.; Werorilangi, S.; Teh, S.J. Anthropogenic debris in seafood: Plastic debris and fibers from textiles in fish and bivalves sold for human consumption. Sci. Rep. 2015, 5, 14340. [CrossRef]

10. Almroth, B.M.C.; Åström, L.; Roslund, S.; Petersson, H.; Johansson, M.; Persson, N.-K. Quantifying shedding of synthetic fibers from textiles; a source of microplastics released into the environment. Environ. Sci. Pollut. Res. 2018, 25, 1191-1199. [CrossRef]

11. Bahners, T.; Ehrler, P.; Hengstberger, M. Erste Untersuchungen zur Erfassung und Charakterisierung textiler Feinstäube. Melliand Textilberichte 1994, 1, 24-30.

12. Wang, Y. Fiber and textile waste utilization. Waste Biomass Valoriz. 2010, 1, 135-143. [CrossRef]

13. Carr, S.A. Sources and dispersive modes of micro-fibers in the environment. Integr. Environ. Assess. Manag. 2017, 13, 466-469. [CrossRef] [PubMed]

14. Browne, M.A.; Crump, P.; Niven, S.J.; Teuten, E.; Tonkin, A.; Galloway, T.; Thompson, R. Accumulation of microplastic on shorelines woldwide: Sources and sinks. Environ. Sci. Technol. 2011, 45, 9175-9179. [CrossRef] [PubMed]

15. Hernandez, E.; Nowack, B.; Mitrano, D.M. Polyester textiles as a source of microplastics from households: A mechanistic study to understand microfiber release during washing. Environ. Sci. Technol. 2017, 51, 7036-7046. [CrossRef] [PubMed]

16. Napper, I.E.; Thompson, R.C. Release of synthetic microplastic plastic fibres from domestic washing machines: Effects of fabric type and washing conditions. Mar. Pollut. Bull. 2016, 112, 39-45. [CrossRef]

17. Sillanpää, M.; Sainio, P. Release of polyester and cotton fibers from textiles in machine washings. Environ. Sci. Pollut. Res. 2017, 24, 19313-19321. [CrossRef]

18. Hartline, N.L.; Bruce, N.J.; Karba, S.N.; Ruff, E.O.; Sonar, S.U.; Holden, P.A. Microfiber masses recovered from conventional machine washing of new or aged garments. Environ. Sci. Technol. 2016, 50, 11532-11538. [CrossRef]

19. Cesa, F.S.; Turra, A.; Baruque-Ramos, J. Synthetic fibers as microplastics in the marine environment: A review from textile perspective with a focus on domestic washings. Sci. Total Environ. 2017, 598, 1116-1129. [CrossRef]

20. Mintenig, S.; Int-Veen, I.; Löder, M.G.; Primpke, S.; Gerdts, G. Identification of microplastic in effluents of waste water treatment plants using focal plane array-based micro-Fourier-transform infrared imaging. Water Res. 2017, 108, 365-372. [CrossRef]

21. Ziajahromi, S.; Neale, P.A.; Rintoul, L.; Leusch, F.D. Wastewater treatment plants as a pathway for microplastics: Development of a new approach to sample wastewater-based microplastics. Water Res. 2017, 112, 93-99. [CrossRef]

22. De Falco, F.; Gullo, M.P.; Gentile, G.; Di Pace, E.; Cocca, M.; Gelabert, L.; Brouta-Agnésa, M.; Rovira, A.; Escudero, R.; Villalba, R. Evaluation of microplastic release caused by textile washing processes of synthetic fabrics. Environ. Pollut. 2018, 236, 916-925. [CrossRef] 
23. Jönsson, C.; Levenstam Arturin, O.; Hanning, A.-C.; Landin, R.; Holmström, E.; Roos, S. Microplastics shedding from textiles-Developing analytical method for measurement of shed material representing release during domestic washing. Sustainability 2018, 10, 2457. [CrossRef]

24. Pirc, U.; Vidmar, M.; Mozer, A.; Kržan, A. Emissions of microplastic fibers from microfiber fleece during domestic washing. Environ. Sci. Pollut. Res. 2016, 23, 22206-22211. [CrossRef] [PubMed]

25. Zeng, E.Y. Microplastic Contamination in Aquatic Environments: An Emerging Matter of Environmental Urgency; Elsevier: Amsterdam, The Netherlands, 2018.

26. Covernton, G.A.; Pearce, C.M.; Gurney-Smith, H.J.; Chastain, S.G.; Ross, P.S.; Dower, J.F.; Dudas, S.E. Size and shape matter: A preliminary analysis of microplastic sampling technique in seawater studies with implications for ecological risk assessment. Sci. Total Environ. 2019, 667, 124-132. [CrossRef]

27. Mellin, P.; Jönsson, C.; Åkermo, M.; Fernberg, P.; Nordenberg, E.; Brodin, H.; Strondl, A. Nano-sized by-products from metal 3D printing, composite manufacturing and fabric production. J. Clean. Prod. 2016, 139, 1224-1233. [CrossRef]

28. Kothari, V. Polyester and polyamide fibres-apparel applications. In Polyesters and Polyamides; Woodhead: Cambridge, UK, 2008; pp. 419-440.

29. De Silva, R.; Wang, X.; Byrne, N. Recycling textiles: The use of ionic liquids in the separation of cotton polyester blends. RSC Adv. 2014, 4, 29094-29098. [CrossRef]

30. Yu, W.; Hancock, B.C. Evaluation of dynamic image analysis for characterizing pharmaceutical excipient particles. Int. J. Pharm. 2008, 361, 150-157. [CrossRef]

31. Zhao, B.; Wang, J. 3D quantitative shape analysis on form, roundness, and compactness with $\mu \mathrm{CT}$. Powder Technol. 2016, 291, 262-275. [CrossRef]

32. Karam, M.C.; Hosri, C.; Hussain, R.; Barbar, R.; Gaiani, C.; Scher, J. Effect of whey powder rehydration and dry-denaturation state on acid milk gels characteristics. J. Food Process. Preserv. 2017, 41, e13200. [CrossRef]

33. Mishra, S. A Text Book of Fibre Science and Technology; New Age International: New Delhi, India, 2000.

34. Dekiff, J.H.; Remy, D.; Klasmeier, J.; Fries, E. Occurrence and spatial distribution of microplastics in sediments from Norderney. Environ. Pollut. 2014, 186, 248-256. [CrossRef] [PubMed]

35. Zambrano, M.C.; Pawlak, J.J.; Daystar, J.; Ankeny, M.; Cheng, J.J.; Venditti, R.A. Microfibers generated from the laundering of cotton, rayon and polyester based fabrics and their aquatic biodegradation. Mar. Pollut. Bull. 2019, 142, 394-407. [CrossRef] [PubMed]

36. Özdil, N.; Kayseri, G.Ö.; Mengüç, G.S. Analysis of abrasion characteristics in textiles. In Abrasion Resistance of Materials; IntechOpen: Rijeka, Croatia, 2012; pp. 119-146. [CrossRef] 